\title{
SALUTO INIZIALE AL CONVEGNO INTERNAZIONALE
}

\author{
ANTONIO PADOA SCHIOPPA
}

A nome del Presidente, il prof. Giannantonio Sacchi Landriani, che purtroppo oggi pomeriggio non può essere presente, porgo ai relatori e a tutti gli intervenuti il cordiale saluto dell'Accademia all'inizio dei lavori di questo importante Convegno scientifico. Nel diritto, nell'economia, nelle scienze matematiche fisiche e naturali, nella medicina, nella religione l'influenza della cultura dei Lumi è stata profonda e duratura, sino al presente.

Già alcuni anni orsono l'Istituto Lombardo aveva maturato il proposito di organizzare un Convegno sull'illuminismo, anche in considerazione del fatto che di questa grande corrente della cultura moderna, di importanza capitale per la storia non solo europea, la Lombardia insieme con il Regno di Napoli è una delle terre d'origine più rilevanti. Le ricerche storiografiche, fiorentissime anche in questi ultimi anni, testimoniano il ruolo centrale che gli intellettuali lombardi e meridionali hanno svolto con loro scritti, presto noti e diffusi in tutta Europa ed anche al di là dell'Atlantico.

Le difficoltà pratiche che facilmente potete immaginare ci hanno indotto a circoscrivere il tema al settore relativamente meno conosciuto dell'economia nel pensiero degli illuministi lombardi, un tema certamente di grande importanza sul quale alcuni dei protagonisti, a partire da Pietro Verri, hanno dato contributi importantissimi. Da alcuni anni è in corso l'edizione nazionale delle opere di Pietro Verri curata da Carlo Capra, un'iniziativa di grande pregio dal punto di vista scientifico. Anche il magnifico Archivio Verri, generosamente donato dalla famiglia alla Fondazione Mattioli, è ormai liberamente consultabile, è a disposizione degli studiosi e sta rivelando molti tesori. E così pure è ormai molto avanzata l'iniziativa parimenti meritevole dell'edizione 
nazionale di Cesare Beccaria, il nome tra tutti più famoso dell'illuminismo europeo sul terreno della giustizia penale.

Sono lieto di ringraziare in particolare i colleghi e amici Pierluigi Porta e Roberto Scazzieri che insieme ad Alberto Quadrio Curzio hanno disegnato questa impostazione sulla tematica del pensiero economico dell'illuminismo lombardo e italiano. $\mathrm{Ci}$ apprestiamo, tra oggi e domani, ad ascoltare una serie di relazioni tenute da eminenti specialisti di questi studi. Naturalmente intendiamo pubblicare gli atti del Convegno.

A tutti l'augurio di buon lavoro.

Antonio Padoa Schioppa Membro effettivo dell'Istituto Lombardo 